\title{
Analogy between sphere forming ability and stemness of human hepatoma cells
}

\author{
YUSUKE UCHIDA $^{1,2}$, SHINJI TANAKA ${ }^{1}$, ARIHIRO AIHARA $^{1}$, RAMA ADIKRISNA $^{1}$, \\ KENICHIROU YOSHITAKE ${ }^{1}$, SATOSHI MATSUMURA ${ }^{1}$, YUSUKE MITSUNORI ${ }^{1}$, \\ AYANO MURAKATA ${ }^{1}$, NORIO NOGUCHI ${ }^{1}$, TAKUMI IRIE ${ }^{1}$, ATSUSHI KUDO ${ }^{1}$, \\ NORIAKI NAKAMURA ${ }^{1}$, PAUL B. LAI $^{3}$ and SHIGEKI ARII ${ }^{1}$
}

${ }^{1}$ Department of Hepato-Biliary-Pancreatic Surgery and ${ }^{2}$ Faculty of Medicine, Tokyo Medical and Dental University, Tokyo, Japan; ${ }^{3}$ Department of Surgery, Prince of Wales Hospital, Hong Kong, SAR, P.R. China

Received April 20,2010; Accepted June 14, 2010

DOI: 10.3892/or_00000966

\begin{abstract}
Increasing evidence suggests that cancers contain a small subset of cancer-initiating cells, so-called cancer stem cells (CSCs) that are capable of regenerating a tumor after chemoradiation therapy. Sphere forming ability is known to be one of properties of CSCs, but the significance remains unclear. The present study focused on sphere formation of human hepatoma cells in three-dimensional culture in order to evaluate the analogy between sphere forming ability and stemness of cancer cells in vitro. Under three-dimensional culture condition, HepG2, Hep3B and PLC/PRF/5 cells demonstrated the sphere formation while SK-Hep1 and Huh-7 cells did not. The population of G0/G1 phase increased in the spheres compared with the monolayer (67 vs. $38 \%$ ). In spite of no significant difference in stem cell surface markers (CD44, CD90, CD133, EpCAM and ABCG2), remarkable upregulation of p27 CDK inhibitor was observed in sphere forming cells. Immunofluorescence analysis revealed the nuclear expression of p27 in the whole of the sphere, but weak expression of p21 only at the peripheral area. The spheres acquired chemoresistance to cisplatin compared with the monolayers (58.9 vs. $16.2 \mu \mathrm{M}$ in IC50). This model was
\end{abstract}

Correspondence to: Dr Shinji Tanaka, Department of HepatoBiliary-Pancreatic Surgery, Tokyo Medical and Dental University, Graduate School of Medicine, 1-5-45 Yushima, Bunkyo-ku, Tokyo 113-8519, Japan

E-mail: shinji.msrg@tmd.ac.jp

Abbreviations: CSCs, cancer stem cells; EpCAM, epithelial cell adhesion module; ABCG2, ATP-binding cassette sub-family G member 2; CDK, cyclic-dependent kinase; IC50, half-maximal inhibitory concentration; PBS, phosphate-buffered saline; PFA, paraformaldehyde; DMSO, dimethyl sulfoxide; SD, standard deviation

Key words: sphere, cancer stem cells, p27, p21, chemoresistance, hepatoma useful for assessment of the role of cell-cycle quiescence in the stemness and chemoresistance of cancer cells.

\section{Introduction}

There is an emerging concept of cancer stem cells (CSCs) that cancer cells do not consist of homogeneous population but include a small subpopulation of cells having the ability of self-renewal and differentiation into multiple phenotypes (1). Several unique properties of CSCs have been identified including drug-efflux ability (side population) $(2,3)$, maintenance of quiescence, sphere formation, high tumorigenicity, and resistance to hypoxia and chemoradiation (4). After anticancer treatment that kills most cancer cells, such drugresistant CSCs might survive and finally generate new populations, resulting in cancer recurrence and metastasis. Detailed analysis on biological characteristics of CSCs are required to overcome the resistance of cancer.

Recent studies revealed that the sphere formation might be essential for cancer-initiating ability of CSCs (5-7), but its significance and mechanism remain unclear. The sphereforming cells in human hepatoma were reported to associate with the expression of stemness markers such as CD90 $(8,9)$, CD133 (10,11), EpCAM (12), ABCG2 (13) and CD44 (14). In this study, we focused on the sphere formation ability using $3 \mathrm{D}$ culture system to evaluate the in vitro analogy to the stemness phenotypes in human hepatoma cells.

\section{Materials and methods}

Cell lines. Human hepatoma cell lines, HepG2, Huh-7, PLC/ $\mathrm{PRF} / 5$ and SK-Hep1 were analyzed (15). Additionally, we used p53-deficient hepatoma cell line Hep3B as well as Hep3B transfected with wild-type p53 gene (16); named as Hep3B-p53(-) and Hep3B-p53(+), respectively. Culture media were the recommended media supplemented with $10 \%$ fetal bovine serum, $100 \mathrm{U} / \mathrm{ml}$ penicillin and $100 \mu \mathrm{g} / \mathrm{ml}$ streptomycin. All cell lines were cultivated in a humidified incubator at $37^{\circ} \mathrm{C}$ in $5 \% \mathrm{CO}_{2}$. Conventional monolayer culture is referred to as two-dimensional culture (2D culture) hereafter in this report. 
Three-dimensional culture (3D culture). Sphere formations were initiated by 3D culture system using low attachment plate (NanoCulture Plate; SCIVAX, Kanagawa, Japan) (17). About $5 \times 10^{3}$ cells were seeded on each well of a 96-well plate. The culture media were the same as that of $2 \mathrm{D}$ culture.

Cell cycle analysis. For cell cycle analysis, spheres were collected by gentle centrifugation $(800 \mathrm{rpm})$ and dissociated mechanically and enzymatically (for $10 \mathrm{~min}$ in trypsin). The single cells were fixed in $70 \%$ ethanol at $4^{\circ} \mathrm{C}$ for $12 \mathrm{~h}$. Cells were washed twice with PBS and stained with propidium iodide at $37^{\circ} \mathrm{C}$ for $30 \mathrm{~min}$. Cell cycle analysis was performed on FACS Calibur flow cytometer (Becton-Dickinson, San Jose, CA, USA).

Protein expression analysis. Total protein was extracted from 2D cultured cells and sphere forming cells at days 3 and 6 (not dissociated) using cell lysis buffer. Western blot analysis was performed using antibodies for CD44/HCAM, p27, p53 (Santa Cruz Biotechnology, Santa Cruz, CA, USA), p21 (Cell Signaling Technology, Beverly, MA, USA), ABCG2/ BRCP (Abcam, Cambridge, UK), CD90 (Abgent, San Diego, CA, USA), EpCAM (AbD Serotec, Oxford, UK) and anti- $\alpha-$ tubulin (Sigma-Aldrich, St. Louis, MO, USA). For analysis of CD133 expression, cells were labeled with anti-CD133 antibody (Miltenyi Biotec, Bergisch Gladbach, Germany), followed by assays on a FACS Calibur flow cytometer (Becton-Dickinson), as previously described (15).

Immunofluorescence analysis. Spheres were cryoembedded in OCT compound (Sakura Finetek, Tokyo, Japan) and sectioned at $10 \mu \mathrm{m}$ thickness with microtome. The sections were fixed in $4 \%$ PFA at $4^{\circ} \mathrm{C}$ for $10 \mathrm{~min}$ and washed three times with distilled water, then stained with an automated immunostainer (BenchMark XT; Ventana Medical Systems, Tucson, AZ, USA). Fluorescence microscopy was performed using Axio Observer (Carl Zeiss, Oberkochen, Germany) and images were acquired digitally using AxioVision (Carl Zeiss).

Chemosensitivity test. For 2D culture, cells were seeded onto a 96-well plate at a density of $2 \times 10^{3}$ cells per well and incubated for $24 \mathrm{~h}$. For 3D culture, cells were seeded on to a 96-well NanoCulture Plate (SCIVAX) at a density of $5 \times 10^{3}$ per well and incubated for 72 or $144 \mathrm{~h}$ (referred to as sphere day 3 and sphere day 6 , respectively). Then, cells were exposed to DMSO control and different concentrations of cisplatin (0.01-300 $\mu \mathrm{M}$; provided by Bristol-Myers Squibb, Tokyo, Japan) for $72 \mathrm{~h}$. As chemosensitivity test, CellTiterGLO luminescence Cell Viability Assay (Promega, Madison, WI, USA) was adopted. CentroLB 960 (Berthold, Bad Wildbad, Germany) was used for recording luminescence.

\section{Results}

Sphere formation ability of hepatoma cell lines. It is known that cells seeded onto NanoCulture Plate grow and aggregate three-dimensionally and finally form spheres. It depends on characteristics of cell lines whether they can form spheres or grow like 2D culture. In HepG2, Hep3B-p53(-), Hep3B$\mathrm{p} 53(+)$ and PLC/PRF/5 cell lines, sphere formation was observed at day 3. In contrast, sphere formation was not observed in SK-Hep1 and Huh-7 cell lines (Fig. 1). In Hep3B-p53(-) cells, sphere formation was observed at day 3, but the verge of spheres began collapsing at day 6. Some of them could not keep spherical body and finally spread twodimensionally. HepG2 cell line was best in respect of the efficacy of sphere formation, so we determined to use HepG2 cell line in the sphere analysis.

Accumulation of quiescent cells in the spheres. We analyzed cell cycle of 2D cultured cells and sphere forming cells at days 3 and 6 (Fig. 2). The population of G0/G1 phase was accumulated in sphere forming cells $(67 \%)$ compared with in 2D cultured cells (38\%). At day 6 in sphere forming cells, remarkable decrease of $\mathrm{S}$ phase was observed. These results indicate that the cultivation of cells as spheres inhibits the transition from $\mathrm{G} 1$ to $\mathrm{S}$ phase.

Expression of cell cycle regulators. In cell cycle analysis, we found that the transition form G1 to $\mathrm{S}$ phase was inhibited in sphere forming cells. To search for the factors that govern G1-S phase transition, we analyzed the expression of cell cycle regulators (p27, p21 and p53) in 2D cultured cells and sphere forming cells at days 3 and 6.p27 and p21 are known to be $\mathrm{CDK}$ inhibitors, which regulate $\mathrm{G} 1$ to $\mathrm{S}$ phase transition by binding to CDKs, and beseids, the expression of p21 is tightly controlled by p53. In addition to those cell cycle regulators, we analyzed the expression of cell surface markers specific for CSCs (ABCG2, EpCAM, CD44, CD90 and $\mathrm{CD} 133)$ to investigate the correlation between spheres and CSCs. In Western blot analysis (Fig. 3A), up-regulation of p27 protein level was observed in sphere forming cells at day 6 , but in contrast, p53 and p21 protein levels were downregulated. No significant difference in hepatocellular carcinoma stem/progenitor cell surface markers was found between 2D cultured cells and sphere forming cells. By immunocytofluorescence of spheres (Fig. 3B), interestingly, the expression of p27 was localized in the nucleus, but the expression of p21 was localized in cells at the peripheral area of spheres.

Sphere forming cells acquired chemoresistance ability. To analyze whether the cultivation of human hepatoma cells as spheres can affect chemosensitivity, 2D cultured cells and sphere forming cells at days 3 and 6 were exposed to 0.01$300 \mathrm{nM}$ cisplatin or DMSO control for $72 \mathrm{~h}$ (Fig. 4). The chemosensitivity test of cisplatin $(0-300 \mu \mathrm{M})$ did not show a significant change of the IC50 value between the cells of 2D culture and sphere at day 3 . In sphere at day 6 , however, the IC50 value of cisplatin was three folds higher compared with 2D cultured cells.

\section{Discussion}

The present study demonstrated that sphere formation contributes to increase of G0/G1 quiescent cells. In cell cycle analysis, the population of G0/G1 phase was accumulated in sphere forming cells (67\%) compared with in 2D cultured cells $(38 \%)$. We then explored factors in inducing quiescence in sphere forming cells. In sphere forming cells, up-regulation of p27 expression was observed at day 6, and the immuno- 


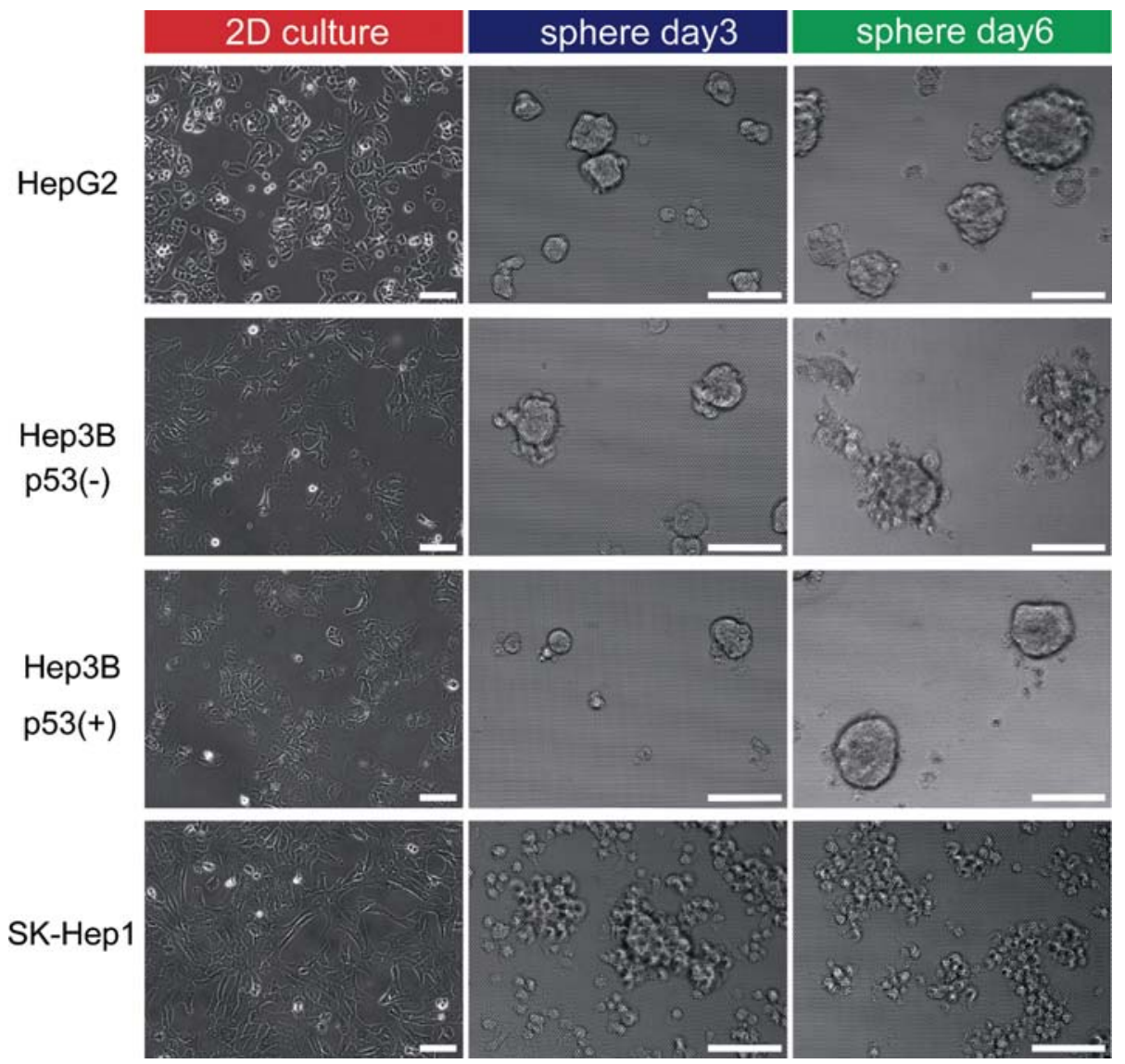

Figure 1. Sphere formation in hepatoma cell lines using 3D culture system. HepG2 cells formed spheres. Hep3B-p53(-) cells formed spheres, but they collapsed at day 6. On the other hand, Hep3B-p53(+) cells formed sphere stably even at day 6. SK-Hep1 cells did not form spheres. Scale bar, $100 \mu \mathrm{m}$.
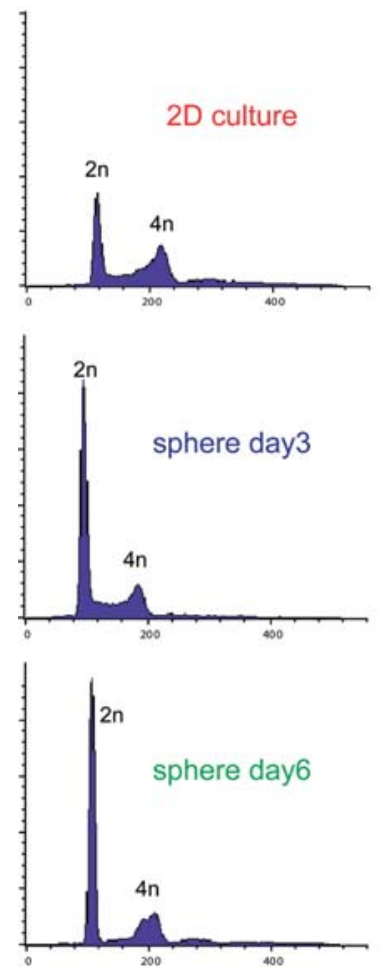

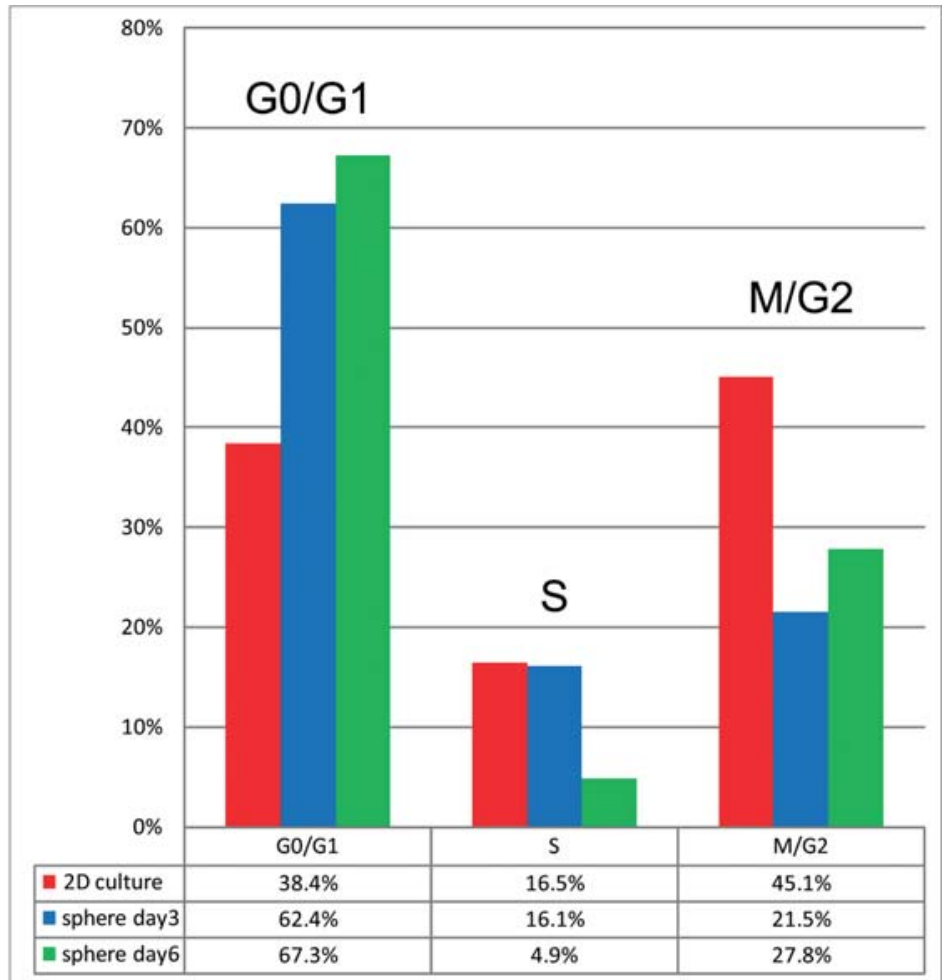

Figure 2. Analysis of HepG2 cell cycle distribution by flow cytometry. The population of G0-G1 phase was increased in sphere forming cells compared with 2D cultured cells. In sphere forming cells at day 6 , a remarkable decrease of the population of S phase was observed. 

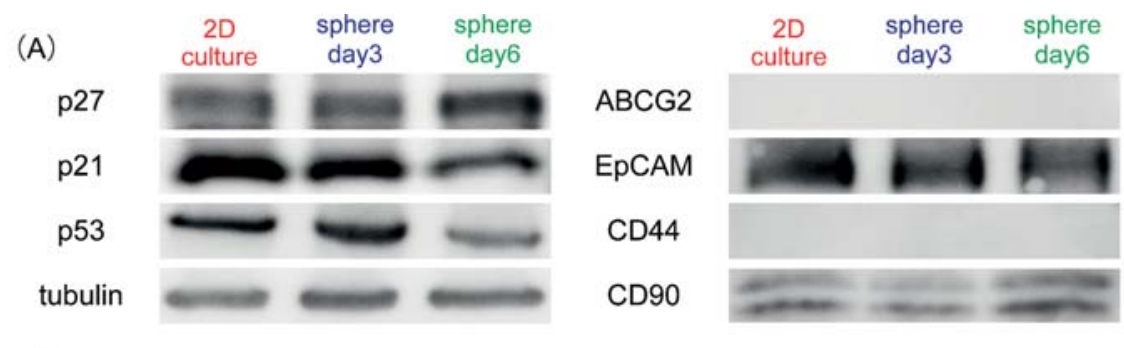

(B)
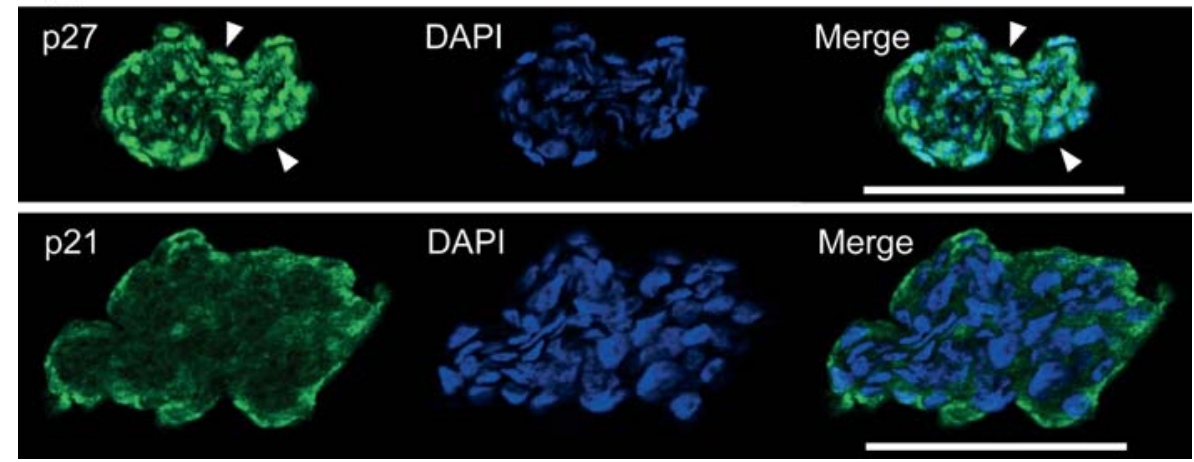

Figure 3. Expression of cell cycle regulators and hepatocellular carcinoma stem/progenitor cell surface markers in spheres of HepG2 cells. (A) Western blot analysis of cell cycle regulator (p27, p21 and p53) and hepatocellular carcinoma stem/progenitor cell surface markers (ABCG2, EpCAM, CD44 and CD90). Tubulin was measured as control. (B) p27 and p21 distribution in HepG2 sphere frozen section. p27 and p21 protein are stained green. The nuclei are stained blue. Scale bar, $100 \mu \mathrm{m}$.

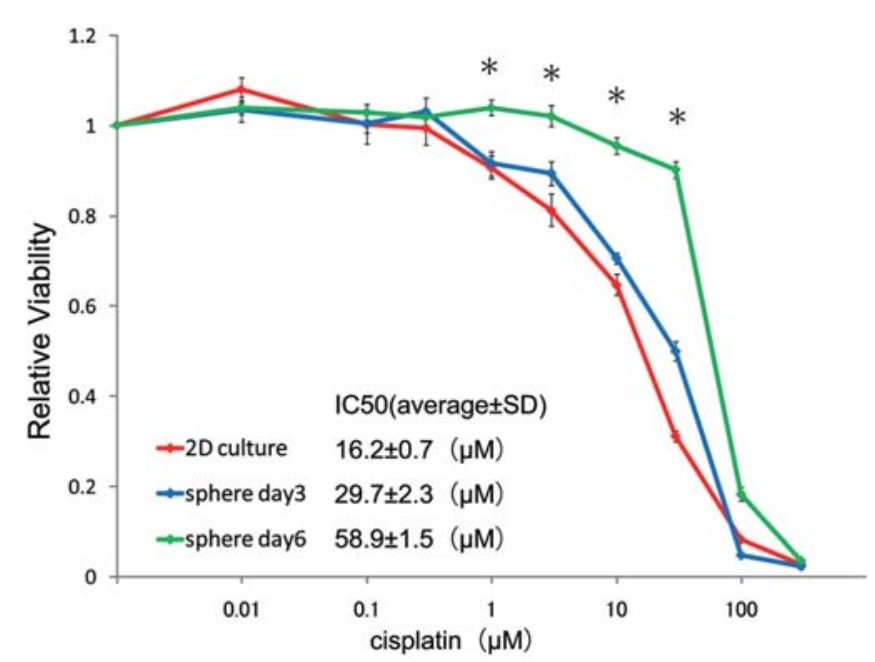

Figure 4. The dose response curve of cisplatin $(0-300 \mu \mathrm{M})$ in HepG2 cells of $2 \mathrm{D}$ culture and sphere at days 3 and 6 . Cells were treated with DMSO control or 0.01-300 nM cisplatin. Data represent relative cell viability of each dose when compared with that of DMSO control. Data are representative of three independent experiments. Statistical analysis was performed using Student's t-test. "P $<0.05$; between $2 \mathrm{D}$ cultured cells and sphere at day 6.

cytofluorescence analysis revealed the nuclear localization of p27. The intranuclear p27 was reported to modulate G1-S phase transition by regulating the activity of CDKs $(18,19)$. It is supposed that p27 in nucleus binds to cyclin E-CDK2 complex and inactivates the complex in G0/G1 phase, but once p27 is transported to cytosol and degraded, the complex is activated and it triggers the transition from $\mathrm{G} 1$ to $\mathrm{S}$ phase. Our data were consistent with this kinetics of p27, so it was suggested that intranuclear p27 plays a central role for cellcycle quiescence in sphere forming cells (20-22).

On the other hand, the $\mathrm{p} 21$ expression was down-regulated in the sphere forming cells. The CDKs inhibitor p21 functions as the key of stress response and major transcriptional target of tumor suppressor gene $p 53(23,24)$. Therefore, the downregulation of p21 might apparently be inconsistent with the cellular quiescence. As suppressor of tumor proliferation, however, the function of p21 is rather ambiguous compared with $\mathrm{p} 27$. In fact, the poor prognosis and clinical progression of HCC were significantly associated with the lack of p27 but not p21 (25-27). By immunocytofluorescence analysis on the spheres, the expression of p21 was localized in cells at the peripheral area of spheres where comparatively active cell proliferation might occur. Considering the down-regulation of $\mathrm{p} 21$ protein levels, the function of p21 may be different from inhibiting cell proliferation in the spheres.

In addition, spheres of Hep3B-p53(+) cells could keep their shapes longer than those of Hep3B-p53(-) cells. Collapse at the verge of spheres was observed at day 6 in Hep3B-p53(-) cells, but spheres of Hep3B-p53(+) cells could keep spherical bodies even at day 6 . It was suggested that the maintenance of sphere formation should be controlled by p53 status. Recently, the p53-p21 pathway has been reported as obstacles to generate the induced pluripotent stem cells, and this pathway is a hot issue in stem cell investigation $(28,29)$. Our data implicated that p53-p21 pathway might play some essential roles in the sphere formation. In chemosensitivity test, the sphere forming cells acquired significant chemoresistance at day 6 , while there were no significant difference of chemosensitivity between the sphere forming cells at day 3 and 2D cultured cells. It is possible that the difference of chemosensitivity was caused not only by cellular quiescence 
but also by some other factors including p53-p21 pathway. Further studies are required to clarify the functional roles of p21 and p27 in cancer stemness as well as chemo-resistance using our model of sphere formation.

\section{References}

1. Reya T, Morrison SJ, Clarke MF and Weissman IL: Stem cells, cancer and cancer stem cells. Nature 414: 105-111, 2001.

2. Chiba T, Kita K, Zheng Y-W, et al: Side population purified from hepatocellular carcinoma cells harbors cancer stem celllike properties. Hepatology 44: 240-251, 2006.

3. Kamohara Y, Haraguchi N, Mimori K, et al: The search for cancer stem cells in hepatocellular carcinoma. Surgery 144: 119-124, 2008

4. Vermeulen L, Sprick MR, Kemper K, et al: Cancer stem cellsold concepts, new insights. Cell Death Differ 15: 947-958, 2008.

5. Dontu G, Abdallah WM, Foley JM, et al: In vitro propagation and transcriptional profiling of human mammary stem/progenitor cells. Genes Dev 17: 1253-1270, 2003.

6. Mani SA, Guo W, Liao MJ, et al: The epithelial-mesenchymal transition generates cells with properties of stem cells. Cell 133 704-715, 2008

7. Cicalese A, Bonizzi G, Pasi CE, et al: The tumor suppressor p53 regulates polarity of self-renewing divisions in mammary stem cells. Cell 138: 1083-1095, 2009.

8. Yang ZF, Ho DW, Ng MN, et al: Significance of CD90+ cancer stem cells in human liver cancer. Cancer Cell 13: 153-166, 2008.

9. Yang ZF, Ngai P, Ho DW, et al: Identification of local and circulating cancer stem cells in human liver cancer. Hepatology 47: 919-928, 2008

10. Ma S, Lee TK, Zheng B-J, et al: CD133+ HCC cancer stem cells confer chemoresistance by preferential expression of the Akt/ PKB survival pathway. Oncogene 27: 1749-1758, 2008.

11. Yin S, Li J, Hu C, et al: CD133 positive hepatocellular carcinoma cells possess high capacity for tumorigenicity. Int J Cancer 120: 1444-1450, 2007.

12. Yamashita T, Ji J, Budhu A, et al: EpCAM-positive hepatocellular carcinoma cells are tumor-initiating cells with stem/ progenitor cell features. Gastroenterology 136: 1012-1024, 2009.

13. Zen Y, Fujii T, Yoshikawa S, et al: Histological and culture studies with respect to ABCG2 expression support the existence of a cancer cell hierarchy in human hepatocellular carcinoma. Am J Pathol 170: 1750-1762, 2007.

14. Zhu Z, Hao X, Yan M, et al: Cancer stem/progenitor cells are highly enriched in CD133(+) CD44(+) population in hepatocellular carcinoma. Int J Cancer 126: 2067-2078, 2010.
15. Aihara A, Tanaka S, Yasen M, et al: The selective Aurora B kinase inhibitor AZD1152 as a novel treatment for hepatocellular carcinoma. J Hepatol 52: 63-71, 2010.

16. Chi TY, Chen GG, Ho LK, et al: Establishment of a doxycyclineregulated cell line with inducible, doubly-stable expression of the wild-type p53 gene from p53-deleted hepatocellular carcinoma cells. Cancer Cell Int 5: 27, 2005.

17. Yamashita Y, Shimada M, Harimoto N, et al: cDNA microarray analysis in hepatocytes differentiation in Huh 7 cells. Cell Transplant 13: 793-799, 2004

18. Sherr CJ and Roberts JM: CDK ihibitors: positive and negative regulators of G1-phase progression. Genes Dev 13: 1501-1512, 1999.

19. Besson A, Dowdy SF and Roberts JM: CDK inhibitors: cell cycle regulators and beyond. Dev Cell 14: 159-169, 2008.

20. Xing H, Wang S, Hu K, et al: Effect of the cyclin-dependent kinases inhibitor p27 on resistance of ovarian cancer multicellular spheroids to anticancer chemotherapy. J Cancer Res Clin Oncol 131: 511-519, 2005.

21. LaRue KEA, Khalil M and Freyer JP: Microenviromental regulation of proliferation in multicellular spheroids is mediated through differential expression of cyclin-dependent kinase inhibitors. Cancer Res 64: 1621-1631, 2004.

22. Leek RD, Stratford I and Harris AL: The role of hypoxiainducible factor-1 in three-dimensional tumor growth, apoptosis and regulation by the insulin-signaling pathway. Cancer Res 65 : 4147-4152, 2005.

23. Weinberg WC and Denning MF: p21 ${ }^{\mathrm{WAF} 1}$ control of epithelial cell cycle and cell fate. Crit Rev Oral Biol Med 13: 453-464, 2002.

24. Coqueret O: New roles for p21 and p27 cell-cycle inhibitors: a function for each cell compartment? Trends Cell Biol 13: 65-70, 2003.

25. Ito Y, Matsuura N, Sakon M, et al: Expression and prognostic roles of the G1-S modulators in hepatocellular carcinoma: p27 independently predicts the recurrence. Hepatology 30: 90-99, 1999.

26. Tannapfel A, Grund D, Katalinic A, et al: Decreased expression of p27 protein is associated with advanced tumor stage in hepatocellular carcinoma. Int J Cancer 89: 350-355, 2000.

27. Chu IM, Hengst L and Slingerland JM: The CDK inhibitor p27 in human cacner: prognostic potential and relevance to anticancer therapy. Nat Rev Cancer 8: 253-267, 2008.

28. Hong H, Takahashi K, Ichisaka T, et al: Suppression of induced pluripotent stem cell generation by the $\mathrm{p} 53-\mathrm{p} 21$ pathway. Nature 460: 1132-1135, 2009.

29. Kawamura T, Suzuki J, Wang YV, et al: Linking the p53 tumour suppressor pathway to somatic cell reprogramming. Nature 460: 1140-1144, 2009. 